\title{
IL-33/ST2 pathway in a bleomycin-induced pulmonary fibrosis model
}

\author{
JIAO XU ${ }^{1}$, JINXU ZHENG ${ }^{2}$, PING SONG ${ }^{2}$, YANJUAN ZHOU ${ }^{1}$ and SHUHONG GUAN ${ }^{3}$ \\ ${ }^{1}$ Department of Respiratory Medicine, Changzhou Wujin People's Hospital, Changzhou, Jiangsu 213017; \\ ${ }^{2}$ Department of Respiratory Medicine, Affiliated Hospital of Jiangsu University, Zhenjiang, Jiangsu 212000; \\ ${ }^{3}$ Department of Respiratory Medicine, The Third Affiliated Hospital of Soochow University, \\ The First People's Hospital of Changzhou, Changzhou, Jiangsu 213003, P.R. China
}

Received April 1, 2015; Accepted February 15, 2016

DOI: $10.3892 / \mathrm{mmr} .2016 .5446$

\begin{abstract}
The present study aimed to investigate the interleukin (IL)-33/ST2 pathway in a model of acute pulmonary fibrosis, and to examine the pathogenesis of pulmonary fibrosis. The pulmonary fibrosis model was established by a single exposure to bleomycin (BLM group) endotracheally to represent idiopathic pulmonary fibrosis, and a control (Cont) group was treated with the same volume of saline. The degrees of acute injury, inflammation and fibrosis were detected using hematoxylin and eosin and Masson's staining. The IL-33, ST2, myeloid differentiation primary response 88 (MyD88) and tumor necrosis factor receptor-associated factor 6 (TRAF6) proteins were detected using Western blotting. The serum levels of IL-4 and IL-13 were detected using an enzyme-linked immunosorbent assay. The results indicated that, compared with the Cont group, there were significant differences in the alveolitis scores in the BLM group on days 3, 7, 14 and $28(\mathrm{P}<0.01)$. The grades of fibrosis were also significantly different on days 7,14 and $28(\mathrm{P}<0.01)$. On examining the dynamic protein expression levels of IL-33, ST2, MyD88 and TRAF6, the expression of IL-33 in the BLM group increased initially, and then decreased gradually following a peak on day 7 . The significant differences between the BLM and Cont groups were observed on days 3 and $7(\mathrm{P}<0.05)$. Compared with the Cont group, the protein levels of ST2, MyD88 and TRAF6 in the BLM group exhibited an increasing trend from day 3, with significant differences, compared with the Cont group, on days 3, 7, 14 and $28(\mathrm{P}<0.05)$. On examination of the serum levels of IL-4 and IL-13 in each
\end{abstract}

Correspondence to: Professor Shuhong Guan, Department of Respiratory Medicine, The Third Affiliated Hospital of Soochow University, The First People's Hospital of Changzhou, 185 Juqian Street, Changzhou, Jiangsu 213003, P.R. China

E-mail: guanshcz@yeah.net

Dr Yanjuan Zhou, Department of Respiratory Medicine, Changzhou Wujin People's Hospital, 2 North Yongning Street, Changzhou, Jiangsu 213017, P.R. China

E-mail: yanjjzhaou@yeah.net

Key words: interleukin-33/ST2, pathogenesis, pulmonary fibrosis group, the levels of IL-4 and IL-13 in BLM group remained higher from day 7 , with peaks on day 28 , and were significantly different, compared with the Cont group, on days 7, 14 and 28 $(\mathrm{P}<0.05)$. In conclusion, the IL-33/ST2 signaling pathway was found to be involved in the rodent model of pulmonary fibrosis induced by bleomycin.

\section{Introduction}

Idiopathic pulmonary fibrosis (IPF) is a complex disease, which remains to be fully elucidated, and is associated with high mortality and morbidity rates, of which the median survival rate following diagnosis is $2-5$ years $(1,2)$. The number of individuals succumbing to pulmonary fibrosis-associated mortality in the USA has increased by $50 \%$ in just 10 years (1992-2003) (3). Previous findings have identified certain mechanisms involving fibrosis, including transforming growth factor- $\beta$ (TGF- $\beta$ ), Wnt ligands, toll-like receptor-mediated signaling and type 2 immune responses (4-7). Although studies have been performed over decades (5-7), the etiology of the disease remains to be elucidated, the mechanism of fibrosis remains unclear and disease-modifying therapies show only poor efficacy.

Interleukin (IL)-33/ST2 signaling, a novel pathway, has been investigated in several fibrotic diseases, including scleroderma, progressive systemic sclerosis and liver fibrosis, in mice and humans (8-10). In the present study, the IL-33/ST2 signaling pathway was examined in a mouse model of lung fibrosis.

\section{Materials and methods}

Regents and instruments. Bleomycin hydrochloride powder (cat. no. H20040205) was purchased from Nippon Kayaku Co., Ltd. (Toyko, Japan). Rabbit anti-mouse myeloid differentiation primary response 88 (MyD88) polyclonal antibody (cat. no. BA2321; 1:2,000) was purchased from Boster Biotechnology, Co., Ltd. (Wuhan, China). Rabbit anti-mouse TRAF6 polyclonal antibody (cat. no. sc-7221; 1:2,000) was purchased from Santa Cruz Biotechnology, Inc. (Santa Cruz, CA, USA). The remaining antibodies, including goat anti-rabbit horseradish peroxidase (HRP)-conjugated antibody (1:2,000; CW Biotech Co., Ltd., Beijing, China), rabbit anti-mouse IL-33 monoclonal antibody (cat. no. AF3626; 1:2,000; R\&D Systems, Inc. CA, USA) and 
Table I. Comparison of the extent of alveolitis and lung fibrosis.

\begin{tabular}{|c|c|c|c|c|c|c|c|c|}
\hline \multirow[b]{2}{*}{ Group } & \multicolumn{2}{|c|}{ Day 3} & \multicolumn{2}{|c|}{ Day 7} & \multicolumn{2}{|c|}{ Day 14} & \multicolumn{2}{|c|}{ Day 28} \\
\hline & Alveolitis & Fibrosis & Alveolitis & Fibrosis & Alveolitis & Fibrosis & Alveolitis & Fibrosis \\
\hline Cont & $1.00 \pm 0.00$ & $1.00 \pm 0.00$ & $1.00 \pm 0.00$ & $1.00 \pm 0.00$ & $1.00 \pm 0.00$ & $1.00 \pm 0.00$ & $1.00 \pm 0.00$ & $1.00 \pm 0.00$ \\
\hline BLM & $2.40 \pm 0.10^{\mathrm{a}}$ & $1.10 \pm 0.10^{\mathrm{a}}$ & $3.50 \pm 0.10^{\mathrm{a}}$ & $1.70 \pm 0.20^{\mathrm{a}}$ & $2.56 \pm 0.15^{\mathrm{a}}$ & $2.63 \pm 0.31^{\mathrm{a}}$ & $2.20 \pm 0.10^{\mathrm{a}}$ & $3.47 \pm 0.25^{\mathrm{a}}$ \\
\hline
\end{tabular}

Data are expressed as the mean \pm standard deviation. ${ }^{\text {a }}<0.05$ BLM group, vs. Cont group. BLM, bleomycin; Cont, control.

rabbit anti-mouse ST2 polyclonal antibody (cat. no. ab72778; 1:2,000; Abcam, Cambridge, UK), were also used in the present study. Commercial kits, including an enzyme-linked immunosorbent assay (ELISA) kit (Biotech Co., Ltd, Beijing, China) and Masson's kit (Nanjing Jiancheng Bioengineering Institute, Nanjing, China), were also used in the present study. Instruments, including a Typhoon 9400 scanner (GE Healthcare Life Sciences, Bethesda, MD, USA), a Power Transfer system (model 3550; Bio-Rad Laboratories, Inc., Hercules, CA, USA) and microplate reader (EL-311; Bio-TEK, Instruments, Inc), were used for the experimental processes.

Animals. The animals used in the present study were purchased from the Laboratory Animal Center of Jiangsu University (Zhenjiang, China). A total of 40 female Kunming strain mice (6 weeks-old, specific pathogen-free grade), weighing $20 \pm 2 \mathrm{~g}$, were maintained at $25^{\circ} \mathrm{C}$ under a 12 -h light/dark cycle with ad libitum access to rodent chow and water. All experiments were performed in accordance with the research proposal for the Care and Use of Laboratory Animals formulated by the Ministry of Science and Technology of China (11). The present study was approved by the ethics committee of the First People's Hospital of Changzhou (Changzhou, China).

Induction of lung injury and pulmonary fibrosis by BLM and sample harvest. Using a random digit table, the 40 mice were evenly divided into two groups: Control group (Cont group, $\mathrm{n}=20$ ) and experimental group (BLM group; $\mathrm{n}=20$ ). On day 0 , following anesthesia by intraperitoneal injection with chloral hydrate $(0.01 \mathrm{mg} / \mathrm{kg}$; Sigma-Aldrich, St. Louis, MO, USA), the BLM group was intratracheally administered bleomycin solution at an optimum dose of $5 \mathrm{mg} / \mathrm{kg}$ (body weight) (12), whereas the animals in the Cont group were administered the same volume of saline. On days 3, 7, 14 and 28 following modeling, five randomly selected mice from each group were sacrificed by cervical dislocation following intraperitoneal injection with $1 \mathrm{mg} / \mathrm{kg}$ pentobarbital sodium (Sigma-Aldrich), and the lung tissues were harvested. The left lung tissues were fixed with $4 \%$ paraformaldehyde (Sigma-Aldrich), whereas the right samples were frozen in liquid nitrogen (Tiangen Biotech Co., Ltd., Beijing, China) for 10 mins and then stored at $-70^{\circ} \mathrm{C}$.

Histopathological assessment of pulmonary injury, inflammation and fibrosis. Following fixing with $4 \%$ paraformaldehyde, the left lung tissues were paraffin-embedded (Tiangen Biotech Co., Ltd.) and $4 \mu \mathrm{m}$ sections were generated. The section were then stained with hematoxylin and eosin to detect the degree of inflammatory injury and Masson's trichrome staining was used to detect the degree of fibrosis. Alveolitis was graded on a scale of 0-3, as follows: 0, normal pulmonary alveolus morphology without alveolar inflammation; 1 , mild alterations, including a widened alveolar septum due to inflammatory cell infiltration; 2 , moderate alterations; and 3, severe alterations, including large numbers of infiltrated inflammatory cells and diffused pathological changes. Pulmonary fibrosis was graded on a scale of $0-3$, as follows: 0 , normal pulmonary tissue without or with only a few filament-like collagen fibers; 1 , slightly increased numbers of collagen fibers showing a fine bundle shape; 2 , moderately increased numbers of collagen fibers that were fused into a narrow-band shape, with a disordered alveolar structure; and 3 , markedly increased numbers of collagen fibers, exhibiting a broad-band or lamellar shape, as well as collapsed or fused pulmonary alveoli and a disordered structure. According to the method established by Szapiel et al (13), scores of 0, 1, 2 and 4 were indicative of grades $0,1,2$ and 3 alveolitis or pulmonary fibrosis, respectively.

Detection of protein expression levels using western blotting. The lung tissues from the two groups were lysed in radioimmunoprecipitation assay buffer (Sigma-Aldrich), which included protease and phosphatase inhibitors. Proteins were extracted using the EpiQuik Whole Cell Extraction kit (cat. no. OP-0003-100; Epigentek Group Inc., Farmingdale, NY, USA) and the protein concentrations were quantified using the BCA kit (cat. no. BCA1-1KT; Sigma-Aldrich). Equal quantities of protein $(10 \mu \mathrm{g})$ were separated by $10 \%$ SDS-PAGE and then transferred onto polyvinylidene fluoride membranes (Tiangen Biotech Co., Ltd.). The membranes were blocked with $5 \%$ skimmed milk powder for $1 \mathrm{~h}$, followed by incubation with primary antibodies for $2 \mathrm{~h}$ at room temperature. After washing the membranes three times for 5 min each with tris-buffered saline containing Tween-20, they were incubated with HRP-conjugated goat anti-rabbit secondary antibody for $1 \mathrm{~h}$ at $37^{\circ} \mathrm{C}$. $\beta$-actin was used as an internal control. Specific bands were visualized using an ECL-PLUS chemiluminescence system (Beyotime Institute of Biotechnology, Wuhan, China) and band intensities were quantified using Land-1D Analyzer software, version 4.0 (Beijing Sage Creation Science, Co., Ltd., Beijing, China).

ELISA for the detection of serum levels of IL-4 and IL-13. Blood samples $(10 \mathrm{ml})$ were obtained from the posterior eyeball veins using heparin-treated glass capillary tubes, and then stored at $-70^{\circ} \mathrm{C}$. To detect the optical density values of IL-4 and IL-13, the supernatants of each group were obtained by centrifugation at $1,000 \mathrm{x} \mathrm{g}$ for $5 \mathrm{~min}$ at $4^{\circ} \mathrm{C}$, and were measured using a 

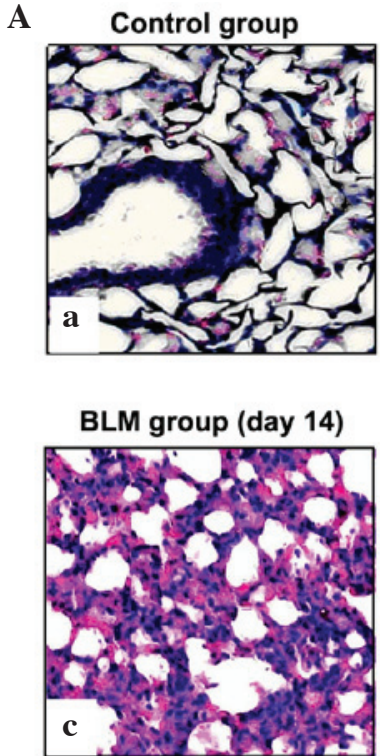

Pathologic changes of lung tissues (H\&E x200)
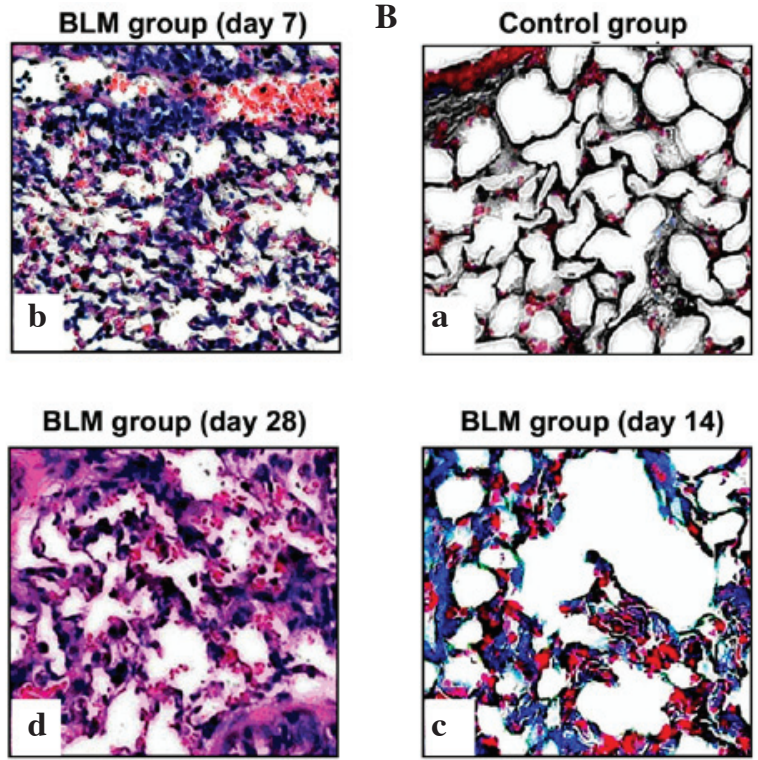
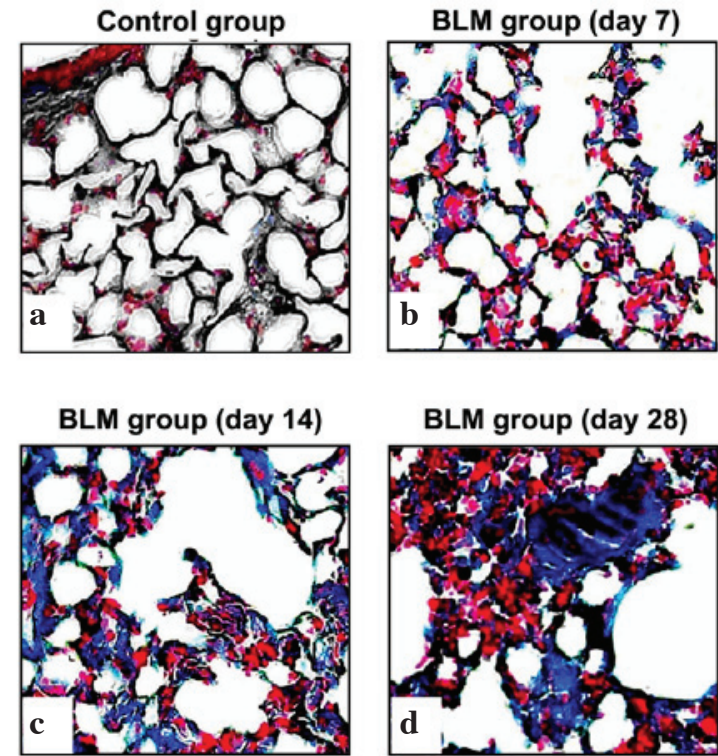

BLM group (day 28)

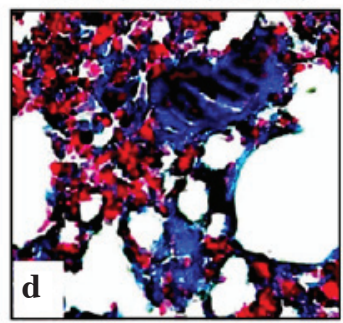

Pathologic changes of lung tissues (Masson x200)

Figure 1. Examination of pathological changes in lung tissues using H\&E and Masson's staining. (A) Examination using H\&E staining. (B) Examination using Masson's staining. (a) control; (b) BLM group day 7; (c) BLM group day 14; (d) BLM group day 28. BLM, bleomycin; H\&E, hematoxylin and eosin.

sandwich ELISA kit, according to the manufacturer's protocol. From a standard curve, the concentrations of each cytokine were obtained.

Statistical analysis. SPSS 16.0 software (SPSS, Inc., Chicago, IL, USA) was used for statistical analyses. Data are presented as the mean \pm standard deviation. Statistical differences between groups were compared using an Independent Sample $t$-test. $\mathrm{P} \leq 0.05$ was considered to indicate a statistically significant difference.

\section{Results}

Pathological changes in lung tissues. The architectures in the lung tissues of the Cont group were normal with no substantial inflammatory cell infiltration or fiber collagen proliferation (Fig. 1Aa and $\mathrm{Ba}$ ). In the BLM group, from day 3 of model establishment, alveolitis began to deepen, with inflammatory cells and erythrocytes in the septations and alveoli, but without evident fiber collagen (Table I). On day 7, the architecture of the alveoli were severely damaged and inflammation peaked, and this was accompanied by a small quantity of fiber collagen (Fig. $1 \mathrm{Ab}$ and $\mathrm{Bb}$ ). The inflammation on day 14 was less severe, however, the alveoli were collapsed and fused, and the septations were widened with extensive fiber collagen (Fig. 1Ac and Bc). On day 28, the inflammation was less marked, however, the architecture of the alveoli were blurred, with wild broad-banded fiber collagen and diffuse lung fibrosis (Fig. 1Ad and Bd). Compared with the Cont group, significant differences were observed in alveolitis on days 3, 7, 14 and 28 ( $\mathrm{P}<0.01)$, and grades of fibrosis were significantly different on days 7,14 and $28(\mathrm{P}<0.01)$.

Protein expression levels of IL-33, ST2, MyD88 and TRAF6. Compared with the Cont group, the protein levels of ST2,
MyD88 and TRAF6 in the BLM group increased from day 3, and reached a peak on day 28 , with significantly higher levels, compared with the Cont group, on days 3, 7, 14 and $28(\mathrm{P}<0.05$; Fig. 2A and B). The expression of IL-33 increased initially, and then decreased gradually with a peak on day 7. Significant differences were observed on days 3 and 7 between the two groups ( $\mathrm{P}<0.05$; Fig. 2A and $\mathrm{B})$.

Serum levels of IL-4 and IL-13. The levels of IL-4 and IL-13 in the serum increased from day 7 and peaked on day 28. The levels were significantly higher, compared with the Cont group, on days 7, 14 and $28(\mathrm{P}<0.05$; Fig. 3).

\section{Discussion}

The present study was the first, to the best of our knowledge, to investigate whether IL-33/ST2 signaling exists in a murine model of bleomycin-induced pulmonary fibrosis. IL-33, as a newly described cytokine of the IL-1 family, can also be termed IL-1F11 according to systematic nomenclature, and has been identified as DVS27, which shows the closest amino acid sequence homology to DVS27 of dogs, and as a nuclear factor from high endothelial venules, which is expressed in the endothelial cell nuclei (14). IL-33 is produced as pro-IL-33, a 31-kDa protein, and is cleaved by caspase- 1 to form a mature $18-\mathrm{kDa}$ protein, which acts as a cytokine through its IL-1-receptor family members $(14,15)$. IL-33, which contributes to the immigration, differentiation and maturation of T helper (Th)2 cells, eosinophils and mast cells, is released passively from damaged epithelial and endothelial cells (16). In the present study, high expression levels of IL-33 were observed during the acute early stage of fibrosis, whereas low expression levels were observed at the late stage of pulmonary fibrosis. This suggested that IL-33, as an alarmin, was secreted from the injured cells induced by bleomycin, and resisted damage and apoptosis (15). 
A

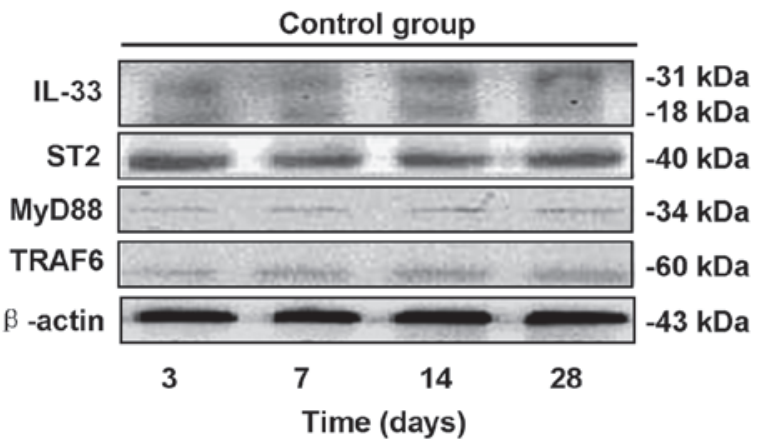

$\mathbf{B}$
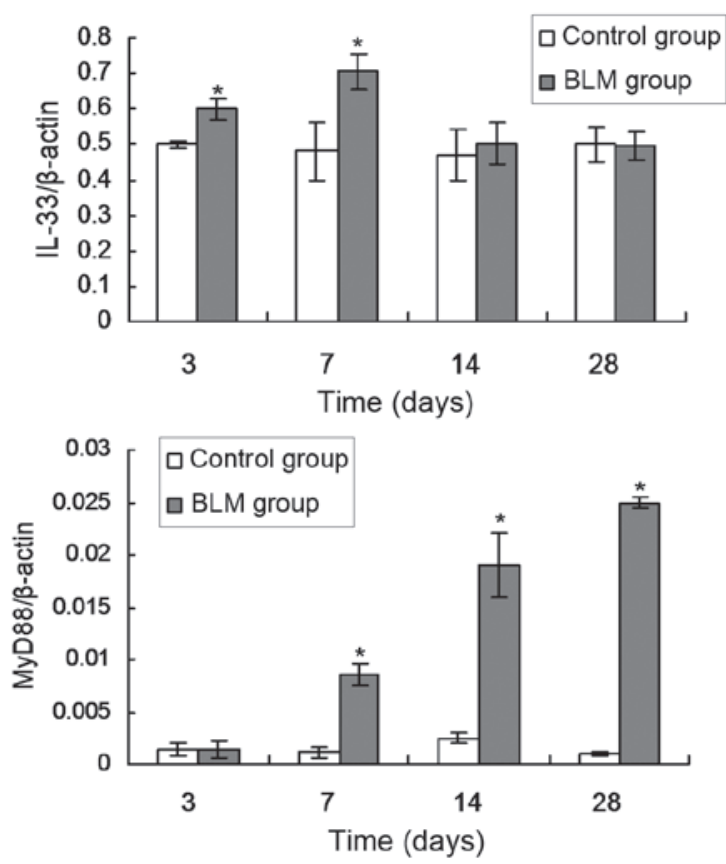
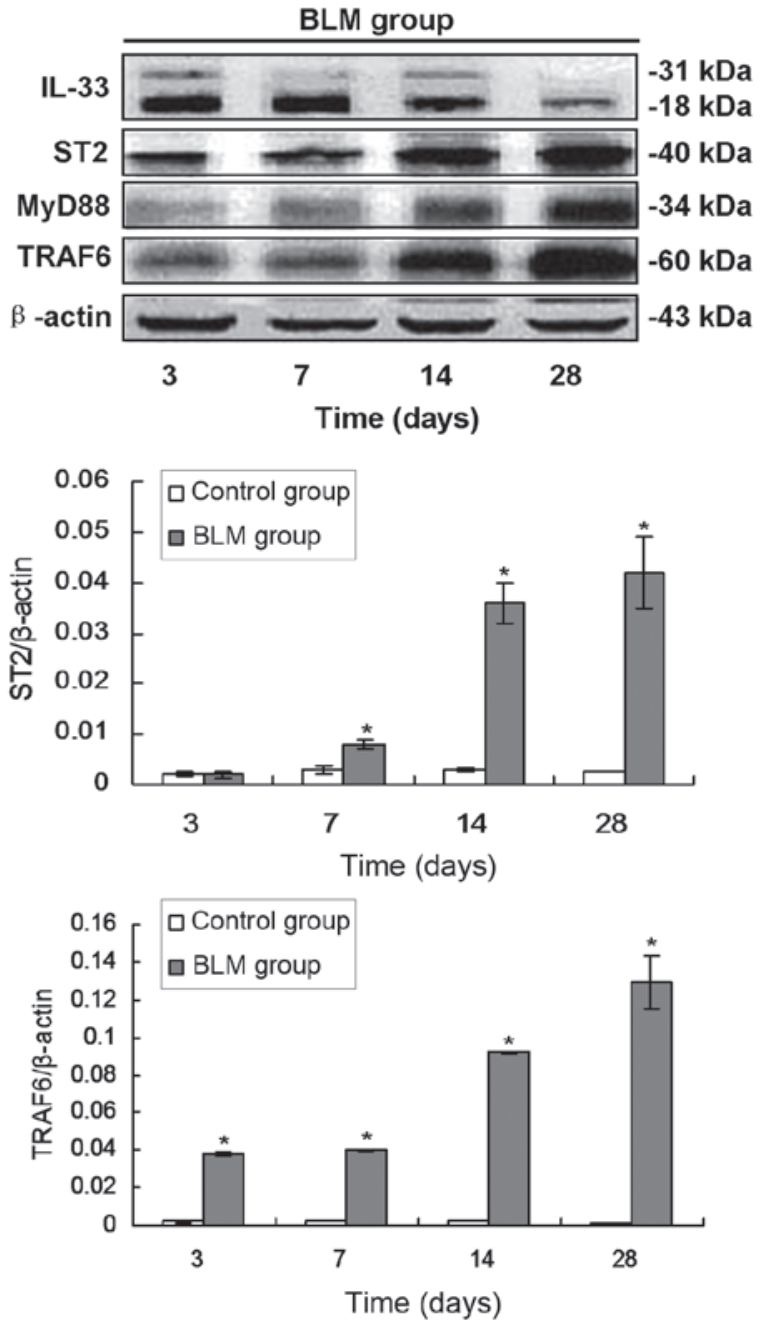

Figure 2. Dynamic protein expression levels of IL-33, ST2, MyD88 and TRAF6 in lung tissues. (A) Western blots for analysis of protein expression. (B) Graphs showing results of statistical analyses to determine the protein expression levels. "P<0.05, vs. control group. BLM, bleomycin; IL-33, interleukin-33; MyD88, myeloid differentiation primary response 88; TRAF6, tumor necrosis factor receptor-associated factor 6.
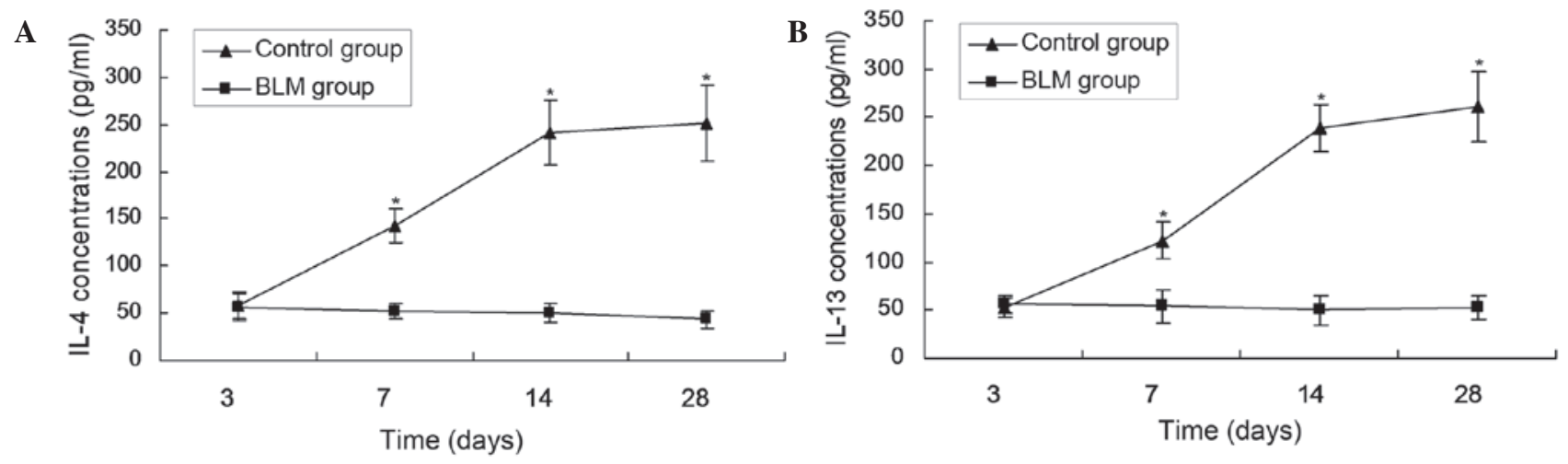

Figure 3. Changes in the serum levels of (A) IL-4 and (B) IL-13 in the control group and BLM group. Data are presented as the mean \pm standard deviation. "P<0.05. compared with the control group. BLM. bleomycin: IL. interleukin.

ST2, the most prominent orphan IL-1 receptor, also termed T1, Fit-1, and DER4, was originally detected as one of the primary response genes at the initial stage of cell proliferation in fibroblasts and belongs to the Toll-like receptor (TLR)-IL-1 receptor superfamily (17-19). Schmitz et al first identified orphan receptor ST2, also termed IL-1R4, as a receptor for
IL-33 via co-immunoprecipitation, and showed that the combination of IL-33 and ST2, through the downstream molecules of ST2, MyD88 and TRAF6, can ultimately lead to the activation of NF-kB and mitogen-activated protein kinases, which are involved in the control of cellular proliferation and apoptosis (14). Xu et al showed that ST2 gene products are 
predominantly expressed in Th2 cells, but not in Th1 cells, and has been recognized as a stable marker of Th 2 cells $(20,21)$. The suggestion that the co-operation of IL-33 and ST2 promotes the activation of Th 2 cells was confirmed by the findings of the present study that the protein expression levels of MyD88 and TRAF6 began to increase from day 3 following model establishment, with a similar trend in the protein level of ST2. This was accompanied by increases in the levels of IL-4 and IL-13, two major cytokines of Th2 cells. Netanya et al confirmed that IL-4 and IL-13 can involve fibrogenesis by upregulating genes associated with wound healing, specifically, arginase, collagens, matrix metalloproteinases (MMPs), and tissue inhibitors of MMP, or by recruiting M2 cells, mast cells, eosinophils, dendritic cells and myofibroblast, facilitating excessive tissue repair and tissue fibrosis $(22,23)$.

Therefore, the present study hypothesized that IL-33, as a 'master switch' of tissue repair that is secreted from dying or apoptotic cells, activated the IL-33-ST2-MyD88-TRAF6 pathway, amplified Th2-type responses and was involved in the pulmonary fibrosis process, via its receptor ST2, a stable marker of Th 2 cells. The pathogenesis of IPF has been described in previous investigations, which revealed that the exuberant deposition of extracellular matrix and the formation of fibrotic foci were induced by the repeated injury of alveolar epithelial cells (24-27). Hayakawa et al (28) showed that soluble ST2 had antagonistic effects on IL-33 signaling, using murine thymoma EL-4 cells stably expressing ST2L and a murine model of asthma. Therefore, intervention of the IL-33/ST2 signaling pathway may offer potential in the therapy of fibrotic diseases, and may be used as a potential target for IPF.

\section{Acknowledgements}

The present study was supported by the Applied Basic Research of Changzhou Municipal Science and Technology Bureau (grant no. CJ20130028).

\section{References}

1. Takenaka K, Gemma A, Yoshimura A, Hosoya Y, Nara M, Hosomi Y, Okano T, Kunugi S, Koizumi K, Fukuda Y, et al: Reduced transcription of the Smad 4 gene during pulmonary carcinogenesis in idiopathic pulmonary fibrosis. Mol Med Rep 2: 73-80, 2009.

2. du Bois RM: Idiopathic pulmonary fibrosis: Present understanding and future options. Eur Respir Rev 20: 132-133, 2011.

3. Olson AL, Swigris JJ, Lezotte DC, Norris JM, Wilson CG and Brown KK: Mortality from pulmonary fibrosis increased in the United States from 1992 to 2003. Am J Respir Crit Care Med 176: 277-284, 2007.

4. Khalil N, O'Connor RN, Flanders KC and Unruh H: TGF-beta 1, but not TGF-beta 2 or TGF-beta 3 , is differentially present in epithelial cells of advanced pulmonary fibrosis: An immunohistochemical study. Am J Respir Cell Mol Biol 14: 131-138, 1996.

5. Villar J, Cabrera N, Valladares F, Casula M, Flores C, Blanch L, Quilez M, Santana-Rodriguez N, Kacmarek R, Slutsky A Activation of the Wnt/b-Catenin signaling pathway by mechanical ventilation is associated with ventilator-induced pulmonary fibrosis in healthy lungs. PLoS One 6: e23914, 2012.

6. Zhang Y,Li Y,Li Y,Li R, Ma Y, Wang H and Wang Y: Chloroquine inhibits MGC803 gastric cancer cell migration via the Toll-like receptor 9/nuclear factor kappa B signaling pathway. Mol Med Rep 11: 1366-1371, 2015.

7. Zhang Z, Chen N, Liu JB, Wu JB, Zhang J, Zhang Y and Jiang X: Protective effect of resveratrol against acute lung injury induced by lipopolysaccharide via inhibiting the myd88-dependent Toll-like receptor 4 signaling pathway. Mol Med Rep 10: 101-106, 2014.
8. Kuroiwa K, Arai T, Okazaki H, Minota S and Tominaga S: Identification of human ST2 protein in the sera of patients with autoimmune diseases. Biochem Biophys Res Commun 284: 1104-1108, 2001

9. Lax A, Sanchez-Mas J, Asensio-Lopez MC, Fernandez-Del Palacio MJ, Caballero L, Garrido IP, Pastor-Perez FJ, Jauzzi JL and Pascual-Figal DA: Mineralocorticoid receptor antagonists modulate galectin-3 and interleukin-33/ST2 signaling in left ventricular systolic dysfunction after acute myocardial infarction. JACC Heart Fail 3: 50-58, 2015.

10. Marvie P, Lisbonne M, L'Helgoualc'h A, Rauch M, Turlin B, Preisser L, Bourd-Boittin K, Théret N, Gascan H, Piquet-Pellorce C and Samson M: Interleukin-33 overexpression is associated with liver fibrosis in mice and humans. J Cell Mol Med 14: 1726-1739, 2010.

11. The Ministry of Science and Technology of the People's Republic of China: Guidance Suggestions for the Care and Use of Laboratory Animals. http://www.most.gov.cn/eng/pressroom/. Accessed September 30, 2006.

12. Tao $\mathrm{Z}$ and Li HP: Comparison of mouse pulmonary fibrosis models induced by bleomycin at different doses. Zhongguo zuzhi gongcheng yanjiu yu linchuang kangfu 13: 1214-1218, 2009 (In Chinese).

13. Szapiel SV, Elson NA, Fulmer JD, Hunninghake GW and Crystal RG: Bleomycin-induced interstitial pulmonary disease in the nude, athymic mouse. Am Rev Respir Dis 120: 893-899, 1979.

14. Schmitz J, Owyang A, Oldham E, Song Y, Murphy E, McClanahan TK, Zurawski G, Moshrefi M, Qin J, Li X, et al: IL-33, an Interleukin-1-like cytokine that signals via the IL-1 receptor-related protein ST2 and induces T helper type 2-associated cytokines. Immunity 23: 479-490, 2005.

15. Baekkevold ES, Roussigné M, Yamanaka T, Johansen FE, Jahnsen FL, Amalric F, Brandtzaeg P, Erard M, Haraldsen G and Girard JP: Molecular characterization of NF-HEV, a nuclear factor preferentially expressed in human high endothelial venules. Am J Pathol 163: 69-79, 2003.

16. Borish L and Steinke JW: Interleukin-33 in Asthma: How big of a role does it play? Curr Allergy Asthma Rep 11: 7-11, 2011.

17. Tominaga S: A putative protein of a growth specific cDNA from BALB/c-3T3 cells is highly similar to the extracellular portion of mouse interleukin 1 receptor. FEBS Lett 258: 301-304, 1989.

18. Yanagisawa K, Tsukamoto T, Takagi T and Tominaga S: Murine ST2 gene is a member of the primary response gene family induced by growth factors. FEBS Lett 302: 51-53, 1992.

19. Bergers G, Reikerstorfer A, Braselmann S, Graninger P and Busslinger M: Alternative promoter usage of the Fos-responsive gene Fit-1 generates mRNA isoforms coding for either secreted or membrane-bound proteins related to the IL-1 receptor. EMBO J 13: 1176-1188, 1994.

20. Xu D, Chan WL, Leung BP, Huang Fp, Wheeler R, Piedrafita D, Robinson JH and Liew FY: Selective expression of a stable cell surface molecule on type 2 but not type 1 helper T cells. J Exp Med 187: 787-794, 1998.

21. Löhning M, Stroehmann A, Coyle AJ, Grogan JL, Lin S, Gutierrez-Ramos JC, Levinson D, Radbruch A and Kamradt T: T1/ST2 is preferentially expressed on murine Th2 cells, independent of interleukin 4, interleukin 5, and interleukin 10, and important for Th2 effector function. Proc Natl Acad Sci USA 95: 6930-6935, 1998.

22. Sandler NG, Mentink-Kane MM, Cheever AW and Wynn TA: Global gene expression profiles during acute pathogen-induced pulmonary inflammation reveal divergent roles for Th1 and Th2 responses in tissue repair. J Immunol 171: 3655-3667, 2003.

23. Rubartelli A and Lotze MT: Inside, outside, upside down: Damage-associated molecular-pattern molecules (DAMPs) and redox. Trends Immunol 28: 429-436, 2007.

24. Tajima S, Oshikawa K, Tominaga S and Sugiyama Y: The increase in serum soluble ST2 protein upon acute exacerbation of idiopathic pulmonary fibrosis. Chest 124: 1206-1214, 2003.

25. du Bois RM: Strategies for treating idiopathic pulmonary fibrosis. Nat Rev Drug Discov 9: 129-140, 2010.

26. Meltzer EB and Noble PW: Idiopathic pulmonary fibrosis. Orphanet J Rare Dis 3: 8, 2008.

27. Selman M, King TE and Pardo A; American Thoracic Society; European Respiratory Society; American College of Chest Physicians: Idiopathic pulmonary fibrosis: Prevailing and evolving hypotheses about its pathogenesis and implications for therapy. Ann Intern Med 134: 136-151, 2001.

28. Hayakawa H, Hayakawa M, Kume A and Tominaga S: Soluble ST2 blocks interleukin-33 signaling in allergic airway inflammation. J Biol Chem 282: 26369-26380, 2010. 